\title{
Analysis of Primary Energy Structure Based on Carbon Emission Reduction Targets and Countermeasures
}

\author{
Feng Ren \\ School of Business and Administration \\ North China Electric Power University \\ Baoding 071003, Hebei Province, China \\ renfeng2002@126.com
}

\begin{abstract}
In order to study the feasibility of China's preset reduction targets in 2030, this study uses linear regression model, grey model and Markov model to forecast China's primary energy consumption, the structure of primary energy consumption and carbon dioxide emissions. This paper analyze the possibility of the completion of China's emission reduction targets in 2030 one by one, and the results show that the nonfossil energy development target which Chinese authorities proposed cannot be achieved without extra effort, and the carbon peak objective which Chinese authorities set cannot be achieved naturally. Hence, this paper give countermeasures and suggestions to help achieve the emission reduction targets in 2030.The results of this study have a certain reference value for the development of China's energy policy.
\end{abstract}

Keywords-Primary energy; Carbon emission reduction; Markov model; GM(1,1) model

\section{INTRODUCTION}

China's carbon emissions and ecological deterioration have attracted more and more attention all over the world. According to the Oslo Norway international climate and Environmental Research Center (CICERO) projections, China's cumulative carbon emissions will exceed the United States in 2016. In 2014, China's total energy consumption reached 42.6 tons of standard coal, accounting for about $1 / 4$ of the global total consumption, and coal consumption accounted for more than half of the world.

The adjustment and optimization of primary energy structure is the core of China's energy strategy for a long period of time in the future. In the past decades, the Chinese government has put more energy into the development of the economy, while ignoring the ecological protection, resulting in the deterioration of the ecological environment. Ecological deterioration is mainly due to the energy structure of high carbon in China. Fortunately, the Chinese government has been aware of the seriousness of the problem, and has begun to focus on energy-saving, emission reduction and ecological protection. The Chinese central government and local governments are making efforts to change this situation. In 2009, China proposed that $\mathrm{CO}_{2}$ emissions per unit of GDP in 2020 should be decreased by $40 \%$ to $45 \%$ than that in 2005 . On 12 November 2014, the Chinese government first formally put forward in the "Sino-US joint statement on climate change" that China's greenhouse gas emissions was expected to reach peak, and the proportion of non-fossil energy in primary energy consumption reached about 20\% by 2030.

China's carbon emission reduction commitment means that the average annual decline rate of carbon intensity during the period of 2005-2030 must be maintained at 3.6\%-4.1\%. Since 1990, however, the average annual decline rates of carbon intensity in some major countries and regions are shown in TABLE I .

TABLE I. THE AVERAGE ANNUAL DECLINE RATES OF CARBON INTENSITY IN TYPICAL COUNTRIES AND REGIONS

\begin{tabular}{c|c|c|c|c}
\hline USA & EU & UK & GER & CHN \\
\hline $2.3 \%$ & $2.3 \%$ & $3.0 \%$ & $2.5 \%$ & $3.6 \%-4.1 \%$ \\
\hline
\end{tabular}

It is estimated that the per capita carbon emissions in China is not more than 10 tons when the amount of carbon emission reaches the peak value, and less than that of the typical developed countries. Carbon emissions per capita in some countries as shown in TABLE II .

TABLE II. PER CAPITA CARBON EMISSIONS IN SOME COUNTRIES (UNIT: TON)

\begin{tabular}{c|c|c|l}
\hline USA & UK & GER & CHN \\
\hline 19.5 & 11.3 & 14.1 & less than 10
\end{tabular}

The analysis above shows that China's commitments about carbon emission reduction targets are worthy of admiration. However, implementation is much more important than commitment. Can China these commitments be achieved? How to optimize the energy structure in the process of implementing the commitment? These are the questions that this paper wants to discuss in depth.

A lot of literatures have been studied on the optimization of primary energy structure in China. Researchers have studied China's commitment to emission reduction targets in 2020 [1- 
7]. For example, Yi, et al believed that, with no additional carbon emission reduction policy, China would be able to achieve emission reduction targets in 2020 [1]. Li, et al believed that in order to achieve the 2020 emission reduction targets, the Chinese government needed to carefully set the economic growth target, while adjusting and optimizing the economic structure and energy structure [6]. The primary energy structure optimization methods can be divided into two kinds, one is focusing on the energy policy, laws and regulations, and the other focused on the quantitative analysis from the aspects of energy substitution, energy intensity and carbon intensity. Based on the scenario analysis method, this paper analyzes the Chinese emission reduction targets, studies the optimization of primary energy structure, and gives the countermeasures and suggestions.

\section{CHINA CARBON EMISSION TARGET ANALYSIS}

If the current energy consumption pattern continues, then the goal of reducing emissions in 2030 can be achieved?

(a) Prediction of energy consumption in 2030. In order to forecast energy consumption in 2030, we establish a linear regression model as follows: $y_{t}=125113+21013.71 t \quad(\mathrm{t}=1,2, \cdots) \quad R^{2}=0.9888 \quad$, $F=1059.89, P=4.46 \times 10^{-13}<0.05$. The test shows that the linear regression equation is significant. According to the regression equation, it can be predicted that the primary energy consumption in 2030 is 75.55 tons of standard coal.

(b) Prediction of primary energy structure. The Markov chain was proposed by Andre Markov in 1906, and has been widely used in natural science; engineering technology and public utility A Markov chain can be expressed as $X_{t}=X(t), t=0,1,2, \cdots$, and has the following property:

$$
\begin{aligned}
& P\left\{X(t+1)=j \mid X(t)=i, X(t-1)=i-1, \cdots, X(1)=i_{1}, X(0)=i_{0}\right\} \\
& =P\{X(t+1)=j \mid X(t)=i\}
\end{aligned}
$$

For Markov chain $\{X(t), t=0,1,2, \cdots\}, P_{i j}(k)$, which is called one-step transition probability, indicates that it is in state $\mathrm{i}$ at time $\mathrm{k}$, and transfers to state $j$ at time $k+1$, i.e.,

$$
P_{i j}(k)=P\{X(k+1)=j \mid X(k)=i\}
$$

For Markov chain $\{X(t), t=0,1,2, \cdots\}, P_{i j}(k, s)$, which is called $k$-step transition probability, indicates that it is in state $i$ at time $k$, and transfers to state $j$ after $k$-step, i.e.,

$$
P_{i j}(k, s)=P\{X(k+s)=j \mid X(k)=i\}
$$

The Markov chain $\{X(t), t=0,1,2, \cdots\} \quad$ is called homogeneous Markov chain, if its one-step transition probability is independent of the state's time, i.e.,

$$
P\{X(k+1)=j \mid X(k)=i\}=P_{i j}
$$

For homogeneous Markov chain, set its absolute probability is $P_{j}(k)$, s-step transition probability is $P_{i j}^{(s)}$, and then the following equations are established:

$$
\begin{gathered}
P_{j}(s+1)=\sum_{i \in I} P_{i}(s) P_{i j} \\
P_{j}(s)=\sum_{i \in I} P_{i}(0) P_{i j}^{(s)}
\end{gathered}
$$

More generally, it is known by the Chapman-Kolmogorov equation, i.e.,

$$
P_{i j}^{(m+n)}=\sum_{k \in I} P_{i k}^{(m)} P_{k j}^{(n)}
$$

These are the theoretical bases of Markov chain prediction.

Based on the historical data of primary energy consumption, the energy structure of China in 2030 is predicted by using Markov model. The transition probabilities between the four kinds of primary energies were calculated as follows by using OLS method:

$$
P=\left[\begin{array}{cccc}
0.7973 & 0 & 0.2027 & 0 \\
0 & 0.5244 & 0 & 0.4756 \\
0.0501 & 0 & 0.9395 & 0.0104 \\
0 & 0.2715 & 0 & 0.7285
\end{array}\right]
$$

On the base of 2014, let $A_{2014}$ denote the vector of the primary energy structure in 2014 and we can formulate the forecasting method as follows:

$$
A_{t}=A_{2014} \times P^{t-2014}
$$

Inputting the values of $A_{2014}=[17.1,6.2,66.0,10.7]$ and $P$ into Formula (9), we can obtain the prediction results: $A_{2030}=[14.91,9.60,57.86,17.63]$. That is to say, by 2030, the proportions of coal, oil, natural gas and non-fossil energy are: $57.86 \%, 14.91 \%$, $9.60 \%$ and $17.63 \%$.

(c) Calculation of carbon dioxide emissions in 2030. Carbon dioxide emission coefficients of coal, oil and natural gas are obtained from the average of the values announced by domestic and foreign authorities (EIA, Japan Institute of energy economics, China Academy of engineering, the global climate change fund, the Asian Development Bank) , respectively 2.6488t/tce, 1.9727 t/tce, 1.5407 t/tce. Energy consumption and carbon dioxide emissions in 2030 are shown in table 3. 
TABLE III. PRIMARY ENERGY CONSUMPTION AND CARBON DIOXIDE EMISSIONS IN 2030 (100 MILLION TONS STANDARD COAL EQUIVALENT)

\begin{tabular}{c|c|c|c|c|c}
\hline & Coal & Oil & Natural gas & Others & Total \\
\hline Energy consumption & 43.71 & 11.26 & 7.25 & 13.33 & 75.55 \\
\hline $\mathbf{C O}_{2}$ emission & 115.79 & 22.22 & 11.17 & 0.00 & 149.18 \\
\hline
\end{tabular}

China's GDP in 2005 was 18.73 trillion Yuan, energy consumption was 23.6 tons of standard coal, and coal, oil, natural gas and non-fossil energy accounted for respectively $78.8 \%, 19.8 \%, 2.6 \%$ and $6.8 \%$. Calculation shows that in 2005 China's carbon emissions were $1.48 \mathrm{Gt}$, equivalent to 54.42 tons of carbon dioxide emissions, which is close to the calculation results of Sun, Liu and other scholars [8-9]. Thus, the carbon intensity in 2005 was 2.9055 tons per ten thousand Yuan.

(d) Analysis of three emission reduction targets.

Target 1: by 2030, carbon dioxide emissions per unit of GDP will drop $60 \%-65 \%$ than that in 2005. Suppose the average annual growth rate of GDP is $r, \frac{149.18}{G D P_{2015} \times(1+r)^{15}}=2.9055 \times 40 \%$, The solution is $r=4.36 \%$. That is to say, if the GDP annual growth rate from 2015 to 2030 is less than $4.36 \%$, then it is impossible to achieve a predetermined carbon intensity target in 2030. (If the intensity of carbon dioxide decreases by 65\%, then the corresponding GDP average annual growth rate is $5.29 \%$.) Given that the global economic recovery is weak, this goal is still more challenging.

Target 2: reach the peak of carbon dioxide emissions by 2030 (120 tons) and try to reach the peak as early as possible. According to the calculation results, China's carbon dioxide emissions in 2030 will reach 149.18 tons, far exceeding the upper limit of China's emissions.

Target 3: non-fossil energy will account for about 20\% in the primary energy consumptions. In the case of no optimization, according to the forecast results, in 2030 nonfossil energy will account for only $17.63 \%$, does not achieve the goal of $20 \%$.

The calculation results show that, if energy consumption patterns are not interfered, China can hardly achieve its own emission reduction targets in 2030.

\section{RECOMMENDATION}

In order to achieve the emission reduction targets in 2030, China must improve the primary energy structure, and must take the road of low carbon energy. Combined with China's national conditions, this paper puts forward the following energy policy recommendations.

(1) China should implement a dual control system for the total amount of carbon emissions and energy consumption in order to control the excessive growth of energy consumption and carbon emissions. Before 2020, China should establish a dual control system of carbon emissions intensity and emissions, develop strict control measures of coal consumption, and effectively curb the excessive growth of energy consumption throughout the country. Energy-saving and energy efficiency improvement are still the main measures for the development of low carbon energies in China. China should establish the long-term mechanism of energy-saving, and further eliminate outdated production capacity in high energy consuming industries; at the same time, China should optimize the industrial structure and improve the technology to promote energy efficiency, especially in the transportation, construction industry, power industry and so on.

(2) China should develop low carbon energies. The low carbon energies need to strive to develop in China, including natural gas, hydropower, nuclear power and non- hydropower renewable energies. (a) Natural gas. Natural gas is one of fossil fuels, with less pollutant emissions compared with coal. In the medium and long term, natural gas will be the fastest growing energy in China's energy structure, and becomes an important force in the development of China's low carbon energies. Unfortunately, China is lack of natural gas. Therefore, China should strengthen international cooperation in the energy field, and look to Africa, Russia, Central Asia, and even Canada as possible alternative sources of its gas imports. (b) Hydropower. Hydropower belongs to non-fossil energy, and it is the most matured and suitable for large-scale development in China. In order to achieve the share of $15 \%$ for non-fossil energy in primary energy, hydropower installed capacity should reach 340 million kW by 2020. (c) Nuclear power. Compared with traditional fossil fuels, nuclear power is a kind of clean energy, without greenhouse gas emissions in its power generation process. Affected by the Fukushima nuclear accident in Japan, China once slowed down the development of nuclear power. In fact, nuclear power in China should be developed vigorously, and constitute a "troika" with natural gas and hydropower together to promote the development of China's low carbon energy. (d) Non-hydropower renewable energies. In the medium-long term, renewable energies such as wind energy, solar energy and biomass energy are important measures to improve the energy structure and reduce the environmental pollution. China should gradually improve the energy law and policies system and create a favorable environment for the development of low carbon energies. China should also promote low carbon energy technology research and development; strengthen the construction of relevant innovation system. Construct national energy science and technology research and development institutions and platforms, in order to accelerate major scientific and technological research, and promote China's low-carbon energy technology to achieve a major breakthrough, as soon as possible to catch up with the pace of global energy technology revolution.

(3) China should change the traditional energy development strategic thinking, and all-round strengthen international cooperation in energy. China energy development strategy in new period should make full use of domestic and foreign resources and markets, with the idea of opening up, from the "self-balancing" gradually transforming to more positive energy "going out" strategy. Currently, the world's leading energy research institutions, including the IEA, have agreed to carbon capture and storage (CCS) technology as the main 
technical means of future carbon emissions reduction. At present, reasons that China's CCS technology is difficult to apply are the high energy consumption and high cost. China should attach great importance to the development of CCS technology, strengthen international cooperation, continuously track the latest progress of CCS technology, and let CCS be more widely used as early as possible.

\section{Conflict of Interests}

The authors declare that there is no conflict of interests regarding the publication of this paper.

\section{ACKNOWLEDGMENT}

Supported by the Fundamental Research Funds for the Central Universities (2015MS150).

\section{REFERENCE}

[1] Yi B, Xu J, Fan Y. Determining factors and diverse scenarios of CO2 emissions intensity reduction to achieve the $40-45 \%$ target by 2020 in China - a historical and prospective analysis for the period 20052020[J]. Journal of Cleaner Production. 2016, 122: 87-101.
[2] Li K, Lin B. China's strategy for carbon intensity mitigation pledge for 2020: evidence from a threshold cointegration model combined with Monte-Carlo simulation methods[J]. Journal of Cleaner Production. 2016, 118: 37-47.

[3] Cansino J M, Román R, Rueda-Cantuche J M. Will China comply with its 2020 carbon intensity commitment?[J]. Environmental Science \& Policy. 2015, 47: 108-117.

[4] Lu Y, Stegman A, Cai Y. Emissions intensity targeting: From China's 12th Five Year Plan to its Copenhagen commitment[J]. ENERGY POLICY. 2013, 61: 1164-1177.

[5] Wang R, Liu W, Xiao L, et al. Path towards achieving of China's 2020 carbon emission reduction target-A discussion of low-carbon energy policies at province level[J]. Energy Policy. 2011, 39(5): 2740-2747.

[6] Li H, Wang L, Shen L, et al. Study of the potential of low carbon energy development and its contribution to realize the reduction target of carbon intensity in China[J]. Energy Policy. 2012, 41: 393-401.

[7] Stern D I, Jotzo F. How ambitious are China and India's emissions intensity targets?[J]. Energy Policy. 2010, 38(11): 6776-6783.

[8] Sun JW, Zhao RQ, Huang XJ, Chen zg. Research on Carbon Emission Estimation and Factor Decomposition of China from 1995 to 2005[J]. Journal of Natural Resources. 2010(8):1284-1295

[9] LIU Qiang, ZHUANG Xing, JIANG Ke-jun, HAN Wen-ke. Energy and Carbon Embodied in Main Exporting Goods of China[J]. China Industrial Economy. 2008(8):46-55 